Research Paper

\title{
HPV test results and histological follow-up results of patients with LSIL Cervical Cytology from the Largest CAP-certified laboratory in China
}

\author{
Baowen Zheng ${ }^{1 *}$, Huaitao Yang ${ }^{2 *}$, Zaibo Li ${ }^{3}$, Guijian Wei ${ }^{1}$, Jia You ${ }^{1}$, Xiaoman Liang ${ }^{1}$, Chengquan Zhao ${ }^{凶}$ \\ 1. Guangzhou Kingmed Diagnostics, Guangzhou, Guangdong 510330, P. R. China; \\ 2. Department of Pathology, University Cincinnati College of Medicine, Cincinnati, OH 45219, USA: \\ 3. Department of Pathology, Wexner Medical Center at Ohio State University, Columbus, OH 43210, USA; \\ 4. Department of Pathology, Magee-Womens Hospital, University of Pittsburgh Medical Center, Pittsburgh, PA 15213, USA. \\ * Both authors made equal contributions to this paper. \\ $\square$ Corresponding author: Chengquan Zhao, MD, Department of Pathology, Magee-Womens Hospital, University of Pittsburgh Medical Center, Pittsburgh, PA \\ 15213, USA. Tel: +412-641-6678, Fax: +412-641-1675, Email zhaoc@upmc.edu
}

(C) Ivyspring International Publisher. This is an open access article distributed under the terms of the Creative Commons Attribution (CC BY-NC) license (https://creativecommons.org/licenses/by-nc/4.0/). See http://ivyspring.com/terms for full terms and conditions.

Received: 2017.01.31; Accepted: 2017.05.30; Published: 2017.07.23

\begin{abstract}
Background: Age-adjusted evaluations have explored the possible utility of (HPV test results in women with LSIL Pap. We investigated HPV test results and histopathologic follow-up results of LSIL patients from China's largest CAP-certified laboratory.

Methods: Patients with LSIL between 2011 and 2015 from the Guangzhou Kingmed Diagnostics were retrospectively retrieved and their hrHPV test results and histological follow-up results were collected and analyzed.

Results: LSIL result was identified in 37,895 cases from 2,206,588 Pap tests (1.7\%) including 1,513,265 liquid-based cytology and 693,323 conventional Pap tests. The average of these women was 38.4 years $(15-88)$. The LSIL reporting rate in women $<30$ years was significantly higher than that in women $>30$ years $(2.1 \%$ vs $1.7 \%)$. The age specific reporting LSIL rates declined with increased age. 8,014 of 37,895 (21.2\%) women with LSIL cytology also had HC2 HPV test results. $75.8 \%$ of women with LSIL Pap tests were hrHPV+ and the HPV+ rates declined with increased age except in patients older than 60 years. Overall histopathologic diagnoses within 6 months after LSIL were identified in 5,987 of 37,895 patients at Guangzhou Kingmed Diagnostics. CIN2/3 was identified in $15.2 \%$ patients, CIN1 in $66.9 \%$, negative in $14.9 \%$ patients. No invasive carcinoma was found in all patients. Of 8014 patients with LSIL Pap test and HPV testing results, 1727 patients had histological follow-up within 6 months after Pap cytology test and HPV testing. The detection rate of CIN2/3 was significantly higher in patients with positive HPV testing result than that in patients with negative HPV testing result (17.8\% vs. $8.1 \%)$. Among patients with LSIL/HPV negative tests, CIN2/3 was detected in 1 of $30(3.3 \%)$ women aged 50 years and above, appearing lower than those in women less than 50 years $(8.0 \%, 28 / 351, \mathrm{P}=0.357)$.

Conclusion: This is the largest histological follow-up study in women with LSIL Pap from China and the data are helpful in establishing a baseline for better understanding the status of cervical screening in China. The 85.1\% positive predict value of LSIL Pap cytology for follow-up CIN lesion was within currently recognized benchmark ranges.
\end{abstract}

Key words: cervical cytology, LSIL, HPV, CIN, Cervical cancer, China.

\section{Introduction}

Cervical cancer remains an important public health problem in Chinese women, especially among women living in rural China. ${ }^{1}$ In contrast to the decreasing incidence trends in developed countries, a substantial increase in cervical cancer incidence was seen in China and it is estimated that 98,900 new cases and 30,500 mortality cases occurred in $2015 . .^{2}$ Most cervical cancer and its precursors are caused by 
persistent high-risk Human Papillomavirus (HPV) infection. While opportunistic cervical screening services are now available in urban areas of China, only during the last decade the prevention services been proposed to gradually widen cervical screening access to rural China. ${ }^{3}$ Currently there is no well-established systematic national cervical cancer registry, no well-organized national cervical screening program, ${ }^{1,3}$ Furthermore, no uniform national standards for cervical cytology quality control exist for laboratories serving this still largely unscreened population. We recently published the Pap cytology reporting profile of China's largest independent laboratory, KingMed Laboratory, which has achieved accreditation through the College of American Pathologists (CAP) and International Laboratory Accreditation Program (LAP), demonstrating that the Bethesda System (TBS) cytology reporting rates from a database of almost 2.37 million Papanicolaou (Pap) test reports were within established CAP benchmark ranges and the histopathologic follow-up cervical intraepithelial neoplasia (CIN) 2+ rates from patients with HSIL cervical cytology results was also within currently recognized benchmark ranges ${ }^{4-6}$.

In current study, we extend the previous findings from Kingmed Diagnostics to analyze HPV test results and histopathologic follow-up results of patients with low-grade squamous intraepithelial lesion (LSIL) cervical cytology.

\section{Materials and Method}

A retrospective study was designed to document LSIL cervical cytology reports from the Guangzhou Kingmed Diagnostics Cytology Laboratory during a period of 5 years from 2011 to 2015. All HPV tests were performed in the CAP accredited Microbiology Laboratory of Kingmed Diagnostics. HPV tests and histological follow-up results were collected and analyzed. Histopathologic tissue procurement procedures included endocervical curettage, cervical biopsy, diagnostic cervical excision by loop electrosurgical excision procedures or cold-knife conization, and hysterectomy.

Four liquid-based cytology (LBC) preparations were used in the KingMed laboratory, including ThinPrep (Hologic, Bedford, Mass), SurePath (BD Diagnostics, Franklin Lakes, NJ), Liqui-PREP (LGM International, Melbourne, FL), and LITUO (Lituo Biotechnology Co., Ltd, Hunan, China). All LBC slides were prepared in the laboratory according to manufacturers' instructions. The Chinese LITUO LBC method is similar to other filtration LBC methods; a sample is fixed in an ethanol based liquid preservative solution and a proprietary filtration method is used for slide preparation. An LTS-YJ2000 automated system (Lituo Biotechnology Co, Ltd, Hunan, China) is used for slide preparation ${ }^{4}$. Conventional Pap tests (CPT) were collected and fixed by individual clinicians and sent to the laboratory for slide preparation and review. Pap test collection methods were largely decided by clinicians.

All Pap tests were reported using the Bethesda System (TBS) 2001 terminology. In this study TBS LSIL terminology refers only to cytology results. Laboratory workload standards, quality control practices, and cytology-histology correlation reviews were all performed in accordance with current CAP LAP checklists. All Pap test screening and final interpretations were performed by a group of around 30 pathologists specialized in gynecologic cytology in the Kingmed Guangzhou Laboratory ${ }^{4-6}$. High-risk HPV (hrHPV) test was performed by using the Hybrid Capture 2 (HC2) test (Qiagen, Hilden, Germany). ${ }^{7}$ The source of hospitals that specimens were collected and the description of Kingmed Guangzhou Laboratory cytology personnel was described in our recent publications. ${ }^{4-7}$

The Pearson chi-square test was used for statistical analysis, which was conducted with SAS 9.1 software (SAS Institute, Cary, NC). A P value less than .05 was considered statistically significant.

\section{Results}

During the 5-year retrospective study period, total 2,206,588 gynecologic cytology reports were identified, including 1,513,265 LBC (68.6\%) and $693,323$ CPTs $(31.4 \%)$ (Table 1$)$. The average age of these women was 38.4 years (15-88). The case numbers increased gradually during the study period, and the annual number of Pap tests reported in 2015 was 2 times more than that reported in 2011 (590,848 versus 299,615 , a $97.2 \%$ increase; Table 1 ).

Table 1. The case number for types of Papanicolaou test by Years.

\begin{tabular}{lllllll}
\hline & ThinPrep & SurePath & LPT & LITUO & CPT & Total \\
\hline 2011 & 157,320 & 22,934 & 25,144 & 8,360 & 85,857 & 299,615 \\
2012 & 143,157 & 34,115 & 25,278 & 16,961 & 118,274 & 337,785 \\
2013 & 152,821 & 42,318 & 25,606 & 31,213 & 157,761 & 409,719 \\
2014 & 171,353 & 28,069 & 0 & 176,812 & 192,387 & 568,621 \\
2015 & 168,189 & 31,600 & 0 & 252,015 & 139,044 & 590,848 \\
Total & 792,840 & 159,036 & 76,028 & 485,361 & 693,323 & $2,206,588$ \\
\hline
\end{tabular}

Abbreviations: ThinPrep, Hologic, Bedford, Massachusetts; SurePath, BD Diagnostics, Franklin Lakes, New Jersey; LPT, Liqui-Prep, LCM International, Melbourne, Florida; LITUO, Lituo Biotechnology Co, Hunan, China; CPT, conventional Papanicolaou test.

The overall LSIL reporting rate was 1.7\% $(37,895)$ among 2206,588 cases of Pap tests. The LSIL reporting rate in LBCs was $2.1 \%(31976 / 1513265)$, significantly higher than $0.9 \%(5919 / 693323)$ in CPT $(p<0.001)$. In 
LBC groups, SurePath showed the highest LSIL reporting rate $(3.3 \%)$ while LPT showed the lowest LSIL rate $(1.5 \%)$. LSIL reporting rate was $2.0 \%$ in ThinPrep preparation, significantly lower than $3.3 \%$ in SurePath preparation $(\mathrm{P}<0.001)$, similar to $2.0 \%$ in LITUO preparation $(\mathrm{p}=0.061)$, and significantly higher than $1.5 \%$ in LPT preparation $(\mathrm{p}<0.001)$ (Table 2). LSIL reporting rates with different preparations were within expected ranges for the CAP 2006 survey benchmarks. ${ }^{8}$

Table 2. LSIL reporting rates in different Pap test preparation methods.

\begin{tabular}{|c|c|c|c|c|}
\hline Cytology & Total Pap & Average age (range) & LISL No (\%) & Average age (range) \\
\hline ThinPrep & 792,840 & $36.47(15-83)$ & $16086(2.0)$ & $38.08(15-89)$ \\
\hline SurePath & 159,036 & $35.99(16-88)$ & $5171(3.3)$ & $37.43(16-80)$ \\
\hline LPT & 76,028 & $35.00(16-77)$ & $1104(1.5)$ & $37.58(16-79)$ \\
\hline LITUO & 485,361 & $41.49(15-78)$ & $9615(2.0)$ & $43.34(15-84)$ \\
\hline CPT & 693,323 & $41.93(16-78)$ & $5919(0.9)$ & $44.40(16-86)$ \\
\hline Total & $2,206,588$ & $38.43(15-88)$ & $37895(1.7)$ & $40.57(15-89)$ \\
\hline
\end{tabular}

Abbreviations: ThinPrep, Hologic, Bedford, Massachusetts; SurePath, BD Diagnostics, Franklin Lakes, New Jersey; LPT, Liqui-Prep, LCM International, Melbourne, Florida; LITUO, Lituo Biotechnology Co, Hunan, China; CPT, conventional Papanicolaou test; LSIL, low-grade squamous intra-epithelial lesion; No, number; Pap test, Papanicolaou test.

\section{Age-specific LSIL reporting rates and HPV-positive rates}

The LSIL reporting rates in different age-specific groups were summarized in Table 3 . The age specific reporting LSIL rate gradually declined with increased age with the highest report rate $3.6 \%$ in group of women younger than 20 years. If the women were divided into two age groups, LSIL report rate was $2.1 \%(7,419 / 350,627)$ in women $<30$ years, significantly higher than $1.7 \%(28,373 / 1,697,087)$ in women $\geq 30$ years $(p<0.001) .8,014$ of $37,895(21.2 \%)$ women with LSIL cytology also had HC2 HPV test results. A positive hrHPV DNA result was obtained in 6,074 of a total $8,014(75.8 \%)$ women with LSIL Pap tests. The age-stratified hrHPV prevalence analyzed in 10-year intervals and the $95 \%$ CI were shown in table 4 . The hrHPV positive rate was highest in adolescents $(87.5 \%)$. There was a clear decline in the hrHPV prevalence in older groups. When women were divided into 2 group groups (younger and older) based on the different cut points of 30, 40 and 50 years, the hrHPV positive rate was significantly higher in all of the younger groups than in the older age groups $(<30 / \geq 3078.5 \% / 71.4 \%, \mathrm{p}<0.001 ;<40 / \geq 40$ $78.5 \% / 71.4 \%, \quad \mathrm{p}<0.001 ; \quad<50 / \geq 50 \quad 76.2 \% / 71.0 \%$, $\mathrm{p}=0.004)$. HrHPV positive rate appeared higher in women 60 years and older than that in women 50-59 age group, $77.2 \%$ vs $69.8 \%$. However, the difference of hrHPV positive rates between these two age groups showed no statistical significance $(p=0.13)$, which may be caused by the small case numbers in the group of women aged 60 years and above.

Table 3. LSIL reporting rates in different age groups.

\begin{tabular}{lllll}
\hline Age, years & \multicolumn{2}{l}{ Total Pap test No LSIL No } & $\%$ & $95 \%$ CI \\
\hline$<20$ & 14,343 & 517 & 3.6 & $3.3-3.9$ \\
$20-29$ & 336,284 & 6,902 & 2.1 & $2.0-2.1$ \\
$30-39$ & 656,239 & 11,346 & 1.7 & $1.7-1.8$ \\
$40-49$ & 705,667 & 12,651 & 1.8 & $1.8-1.8$ \\
$50-59$ & 265,011 & 3,542 & 1.3 & $1.3-1.4$ \\
$60-69$ & 62,888 & 751 & 1.2 & $1.1-1.3$ \\
$\geq 70$ & 7,282 & 83 & 1.1 & $0.9-1.3$ \\
Unknown & 158,874 & 2,103 & 1.3 & $1.3-1.4$ \\
Total & $2,206,588$ & 37,895 & 1.7 & $1.7-1.7$
\end{tabular}

Abbreviations: LSIL, low-grade squamous intra-epithelial lesion; No, number; Pap test, Papanicolaou test; CI, confidence Interval.

Table 4. HPV positive rates in women with LSIL Pap in different age groups.

\begin{tabular}{lllll}
\hline Age, years & $\begin{array}{l}\text { HPV tested } \\
\text { No }\end{array}$ & $\begin{array}{l}\text { HPV positive } \\
\text { No }\end{array}$ & 95\% CI \\
\hline$<20$ & 120 & 105 & 87.5 & $80.2-92.8$ \\
$20-29$ & 1933 & 1600 & 82.8 & $81.0-84.4$ \\
$30-39$ & 2653 & 1989 & 75.0 & $73.3-76.6$ \\
$40-49$ & 2333 & 1669 & 71.5 & $69.7-73.3$ \\
$50-59$ & 519 & 362 & 69.8 & $65.6-73.7$ \\
$\geq 60$ & 101 & 78 & 77.2 & $67.9-85.0$ \\
Unknown & 355 & 271 & 76.3 & $71.6-80.7$ \\
Total & 8014 & 6074 & 75.8 & $74.8-76.7$ \\
\hline
\end{tabular}

Abbreviations: LSIL, low-grade squamous intra-epithelial lesion; No, number; $\mathrm{HPV}$, human papillomavirus; CI, confidence Interval.

\section{Histopathologic follow-up results in women with LSIL cytology}

Of 37,895 women with LSIL Pap test results, $5,987(15.8 \%)$ had at least one histopathologic follow-up diagnosis within 6 months after the LSIL Pap test at KingMed laboratory, including 5,342 cases with LBC $(89.2 \%)$ and 645 cases with CPT $(10.8 \%)$ (Table 5). The Kingmed Diagnostics is an independent laboratory and cervical cytology and surgical pathology specimens are received from diverse hospitals. Cases with follow-up surgical pathology at the outside institution were excluded from current study. For most patients, the biopsy represented the first histological follow-up result. If there were 2 or more follow-up histologic results, the more serious lesions were recorded. The average age of the patients with histopathologic follow-up results was 38.4 years. Overall, CIN2/3 lesion was identified in 909 (15.2\%) patients, including 909 of 5,342 patients (15.3\%) with LBC and 93 of 645 patients (14.4\%) with CPT, which was not statistically different $(p=0.095)$.CIN1 lesion was found in $66.9 \%$ of the patients and negative cases in $14.9 \%$ of the patients. No invasive cervical carcinoma diagnoses identified. The CIN2/3 histopathologic follow-up results were compared 
with after LSIL Pap tests by using different cytological preparations. CIN2/3 detection rate in ThinPrep was significantly higher than that in SurePath preparation (16.2\% vs. $13.1 \%, p=0.017)$, but was not significantly different when other cytology preparation methods including CPT were compared with individually with ThinPrep result.

Table 5. Histopathologic follow-up results after LSIL cytology with different Pap test methods.

\begin{tabular}{lllll}
\hline Cytology & $\begin{array}{l}\text { F/u case } \\
\text { No }\end{array}$ & CIN2/3 $(\%)$ & CIN1 (\%) & Negative (\%) \\
& 3,066 & $498(16.2)$ & $2,139(69.8)$ & $429(14.0)$ \\
ThinPrep & 994 & $130(13.1)$ & $720(72.4)$ & $144(14.5)$ \\
SurePath & 155 & $22(14.2)$ & $104(67.1)$ & $29(18.7)$ \\
LPT & 1,127 & $166(14.7)$ & $754(66.9)$ & $207(18.4)$ \\
LITUO & 645 & $93(14.4)$ & $467(72.4)$ & $85(13.2)$ \\
CPT & 5,987 & $909(15.2)$ & $4,184(66.9)$ & $894(14.9)$ \\
Total &
\end{tabular}

Abbreviations: LSIL, low-grade squamous intra-epithelial lesion; Pap test, Papanicolaou test; ThinPrep, Hologic, Bedford, Massachusetts; SurePath, BD Diagnostics, Franklin Lakes, New Jersey; LPT, Liqui-Prep, LCM International, Melbourne, Florida; LITUO, Lituo Biotechnology Co, Hunan, China; CPT, conventional Papanicolaou test; $\mathrm{F} / \mathrm{u}$, follow-up; No, number; $\mathrm{CIN}$, cervical intraepithelial neoplasia.

Of 8014 patients with LSIL Pap test and HPV testing results, 1727 patients had histological follow-up within 6 months after Pap cytology test and HPV testing. The detailed histopathologic follow-up results, correlated with hrHPV test results and stratified by ages in 10-year age group intervals, are present in table 6 . The detection rate of CIN2/3 was significantly higher in patients with positive hrHPV testing compared patients with negative hrHPV testing $(17.8 \%$ vs. $8.1 \%, \mathrm{p}<0.001)$. The overall CIN detection rate was $88.8 \%(1185 / 1334)$ in women with positive hrHPV testing, significantly higher the rate of $84.7 \%$ in women with negative hrHPV testing $(\mathrm{p}=0.029)$. Regardless hrHPV testing results, CIN2/3 detection rate was $11.4 \%(54 / 475)$ in women age less than 30 years, significant lower than $17.4 \%(208 / 1198)$ in women age 30 years and above $(p=0.002)$. in $\mathrm{hrHPV}$ negative group CIN2/3 detection was found in $7.9 \%$ women $(28 / 351)$ age less than 50 years, appearing higher $3.3 \%$ in women aged 50 years and above.
However the difference showed no statistical significance $(p=0.357)$ due to the small case numbers.

\section{Discussion}

The Kingmed laboratory is the largest independent laboratory in China with CAP LAP certification and International Organization for Standardization (ISO) certification. As part of an effort to improve patient safety by meeting or exceeding requirements achieve laboratory standardization according to international standards, Kingmed laboratory established a quality assurance program to analyze its cervical cytology reporting rates and histologic follow up results for patients with abnormal Pap cytology. Recently, the TBS reporting rates for 1,394,389 Pap tests between 2007 and 2012 from Kingmed laboratory have been published and were found to be largely within current CAP benchmark ranges. ${ }^{4}$ Furthermore, the recently published data from Kingmed also demonstrate the positive predict value (PPV) for CIN2/3 diagnoses after HSIL cytology reports fall within available CAP benchmark ranges and the cytology-histology correlation rate in patients with SCC cytology reports well falls in the benchmark range from CAP. ${ }^{5}$ Data presented in the current study further extend prior findings by including LSIL reporting rates in different age groups, HPV-positive rates and histological follow up results from KingMed laboratory. Similar to our previous studies, current study demonstrates the LSIL reporting rate fell into the CAP benchmark ranges and the LSIL reporting rate with LBC was significantly higher than that reported with CPT $(2.1 \%$ VS $0.9 \%$ ), which are consistent with previous observations documented by US CAP LAP laboratories (the median reporting rate for LSIL in ThinPrep $3.0 \%$ vs Conventional 1.3\%). ${ }^{8}$ Among the four LBC methods, the LSIL reporting rate is the highest in SurePath (3.3\%) and lowest in LPT (1.5\%). The different reporting rates can be caused by many factors, such as specimen collection devices and variable populations.

Table 6. Histopathologic follow-up results for women with LSIL/HPV testing results, by different ages.

\begin{tabular}{|c|c|c|c|c|c|c|c|c|}
\hline \multirow[t]{2}{*}{ Age (Years) } & \multicolumn{4}{|c|}{ hrHPV Positive Cases } & \multicolumn{4}{|c|}{ hrHPV Negative Cases } \\
\hline & Follow-up No. & CIN2/3 (\%) & CIN1 (\%) & Negative (\%) & Follow-up No & CIN2/3 (\%) & CIN1 (\%) & Negative (\%) \\
\hline$<20$ & 24 & $2(8.3)$ & $16(66.7)$ & $6(25.0)$ & 3 & $0(0)$ & $3(100)$ & $0(0)$ \\
\hline $20-29$ & 367 & $49(13.4)$ & $284(77.4)$ & $34(9.3)$ & 81 & $3(3.7)$ & $68(84.0)$ & $10(12.3)$ \\
\hline $30-39$ & 440 & $92(20.9)$ & $298(67.7)$ & $50(11.4)$ & 135 & $15(11.1)$ & $100(74.1)$ & $20(14.8)$ \\
\hline $40-49$ & 379 & $75(19.8)$ & $263(69.4)$ & $41(10.8)$ & 132 & $10(7.6)$ & $102(77.3)$ & $20(15.2)$ \\
\hline $50-59$ & 68 & $14(20.6)$ & $46(67.6)$ & $8(11.8)$ & 28 & $1(3.6)$ & $18(64.3)$ & $9(32.1)$ \\
\hline$\geq 60$ & 14 & $1(7.1)$ & $6(42.9)$ & $7(50.0)$ & 2 & $0(0)$ & $2(100)$ & $0(0)$ \\
\hline Unknown & 42 & $5(11.9)$ & $34(81.0)$ & $3(7.1)$ & 12 & $3(25.0)$ & $8(66.7)$ & $1(8.3)$ \\
\hline Total & 1,334 & $238(17.8)$ & $947(71.0)$ & $149(11.2)$ & 393 & $32(8.1)$ & $301(76.6)$ & $60(15.3)$ \\
\hline
\end{tabular}

Abbreviations: LSIL, low-grade squamous intra-epithelial lesion; HPV, human papillomavirus; F/u, follow-up; No, number; CIN, cervical intraepithelial neoplasia. 
Our current data also indicated that LSIL reporting rates declined with increased ages, with the peak in women younger than 20 years. Statistical analysis demonstrated the LSIL reporting rate in women $<30$ years was significantly high than that in women $>30$ years, suggesting the highest prevalence of HPV infection in younger women.

In this study cohort, $75.8 \%(6,074 / 8,014)$ women with LSIL Pap tests had positive HC2 HPV testing results. A large meta-analysis of the HPV test positivity rate of women with LSIL showed that the pooled positivity rate was $76 \% .9^{9}$ The average $\mathrm{HPV}$-positive rate in our cohort is very similar to this pooled rate, slightly lower than the rate reported by ALTS group, ${ }^{10}$ or Kaiser Permanente Northern California $(\mathrm{KPNC})^{11}$, but higher than that from UK ARTISTIC trials. ${ }^{12} \mathrm{HPV}$-positive rate of women aged 30 years and older with LSIL cytology parallels to that from ALTS ${ }^{13}$ and falls between the rated reported by Kaiser Permanente 11 and the rate reported by UK ARTISTIC trials. ${ }^{12}$ The HPV positive rate was higher in young women than that in women of middle age, consistent with the high prevalence of HPV infection in young women. Surprisingly, the HPV positive rate was also higher in women $\geq 60$ years than that in women $40-59$ years.

The $85.1 \%$ PPV (5093/5987) of LSIL Pap cytology for follow-up CIN lesions reported in current study was within currently recognized benchmark ranges for international cytology laboratories. The overall CIN2+ (CIN2 and above lesions) rate of women with LSIL cytology in Kingmed data (15.18\%) is very close to that from KPNC 11 and lower than that from ALTS14 in sub-categorized HPV-positive women with LSIL cytology, but higher than those of UPMC ${ }^{15}$ and Yale's studies ${ }^{16}$. Furthermore, the CIN2+ age-specific rates of HPV-positive women with LSIL cytology from our current study are similar to those from KPNC in mid-age women (30-49 year women). However, the CIN2+ rate was sustained after age 50 years rather than gradually decreased as reported in Castle's KPNC. ${ }^{11,} 17$ The sustained high CIN2+ rate with increased age in current study is probably due to unscreened patients and/or the heterogeneity of patients. Meanwhile, in HPV-negative women with LSIL cytology, the CIN2+ rate in current study $(8.1 \%)$ is higher than that reported from UPMC (3.4\%), 18 Kaiser Permanente Northern California $(4.0 \%)^{11,17}$ and Methodist Hospital of Houston $(1.3 \%, 1 / 77)^{19}$ By another calculation method, $32(11.9 \%)$ women had negative HPV testing among 270 women with LSIL and histologic diagnosis of CIN2/3. Taken together, these results indicate the limitation of HPV testing in women with LSIL and suggest immediate colposcopy may be the best follow up management for women with LSIL.

The data presented in current study together with our previous findings from Kingmed not only demonstrate the advantage of LBC in identifying cervical cytology abnormalities, but also provide an opportunity to compare the performance of different LBC methods, including FDA approved methods (ThinPrep and SurePath) and non-FDA-approved methods (LITUO and Liqui-Prep). The highest documented Kingmed reporting rate for LSIL was with SurePath LBC samples, and the lowest reporting rate was with LPT LBC samples, which are similar to our recently published data regarding HSIL reporting rates. ${ }^{5}$ These differences of LSIL reporting rates among different Pap preparations may also be related to the different populations tested, however, specific reason for the differences cannot be determined with certainty due to the samples from numerous hospitals and different populations. Two LBC preparation methods with cheap price from China were used in this study, the LSIL reporting rates from cases prepared by these two methods were similar to that from cases prepared by ThinPrep, confirming their reliability to detect abnormalities.

In summary, this largest histological follow-up study in women with LSIL Pap from China further extends our previously reported findings from a large CAP-certified independent laboratory using diverse cytology methods and demonstrates the LSIL reporting rate, HPV-positive rate, and the PPV for CIN2/3 diagnoses after LSIL cytology fall within available CAP and UK benchmark ranges and are comparable with other international cytology laboratories. Our data also demonstrated a considerably high CIN2/3 rate in women with LSIL and negative HPV testing, suggesting an intermediate colposcopy would be necessary for these women's management and the limitation with HPV testing for the primary screening. Furthermore, current data are helpful in establishing a baseline for better understanding the status of cervical screening in China.

\section{Competing Interests}

The authors have declared that no competing interest exists.

\section{References}

1. Shi JF, Qiao YL, Smith JS, et al. Epidemiology and prevention of human papillomavirus and cervical cancer in China and Mongolia. Vaccine. 2008; 26 Suppl 12: M53-9.

2. Chen W, Zheng R, Baade PD, et al. Cancer statistics in China. 2014. CA Cancer J Clin. 2016; 66:115-32

3. Li J, Kang LN, and Qian LN. Review of the cervical cancer disease burden in mainland China. Asian Pac J Cancer Prev. 2011;12(5): 1149-53.

4. Zheng B, Austin RM, Liang X, et al. Bethesda System reporting rates for conventional Papanicolaou tests and liquid-based cytology in a large Chinese, 
College of American Pathologists-certified independent medical laboratory: analysis of 1394389 Papanicolaou test reports. Arch Pathol Lab Med. 2015;139(3): 373-7.

5. Zheng B, Austin RM, Liang X, et al. PPV of an HSIL cervical cytology result in China's largest CAP-certified laboratory. Journal of the American Society of Cytopathology. 2014;4(2): 84-89.

6. Zheng B, Li Z, Austin RM, Chen C, Zhao C. Cervical Cytology Reporting Rates from China's Largest College of American Pathologists-Certified Laboratory with a Focus on Squamous Cell Carcinoma Cytology and Its Histopathological Follow-Up Results. Acta Cytol. 2015;59(5):399-404

7. Zeng $Z$, Austin RM, He $X$, et al. Prevalence of High-Risk Human Papillomavirus Infection in China: Analysis of 671,163 Human Papillomavirus Test Results From China's Largest College of American Pathologists-Certified Laboratory. Am J Clin Pathol. 2016;145(5):622-5.

8. Eversole GM, Moriarty AT, Schwartz MR, et al. Practices of participants in the college of american pathologists interlaboratory comparison program in cervicovaginal cytology. 2006. Arch Pathol Lab Med, 2010;134(3): 331-5.

9. Arbyn, M, Martin-Hirsch P, Buntinx F, Van Ranst M, Paraskevaidis E, Dillner J. , Triage of women with equivocal or low-grade cervical cytology results: a meta-analysis of the HPV test positivity rate. J Cell Mol Med, 2009;13(4): 648-59.

10. ALTS, Human papillomavirus testing for triage of women with cytologic evidence of low-grade squamous intraepithelial lesions: baseline data from a randomized trial. The Atypical Squamous Cells of Undetermined Significance/Low-Grade Squamous Intraepithelial Lesions Triage Study (ALTS) Group. J Natl Cancer Inst. 2000; 92(5): 397-402.

11. Littell RD, Kinney W, Fetterman B, et al. Risk of cervical precancer and cancer in women aged 30 years and older with an HPV-negative low-grade squamous intraepithelial lesion screening result. J Low Genit Tract Dis. 2011;15(1): 54-9.

12. Kitchener $\mathrm{HC}$, Almonte $\mathrm{M}$, Gilham $\mathrm{C}$, et al ARTISTIC: a randomised trial of human papillomavirus (HPV) testing in primary cervical screening. Health Technol Assess. 2009;13(51): 1-150, iii-iv.

13. Sherman ME, Schiffman M, and Cox JT. ASCUS/LISL Triage Study Group. Effects of age and human papilloma viral load on colposcopy triage: data from the randomized Atypical Squamous Cells of Undetermined Significance/Low-Grade Squamous Intraepithelial Lesion Triage Study (ALTS). J Natl Cancer Inst. 2002;94(2): 102-7.

14. ALTS. A randomized trial on the management of low-grade squamous intraepithelial lesion cytology interpretations. Am J Obstet Gynecol. 2003;188(6): 1393-400.

15. Heider A,Austin RM, and Zhao C. HPV test results stratify risk for histopathologic follow-up findings of high-grade cervical intra-epithelial neoplasia in women with low-grade squamous intra-epithelial lesion Pap results. Acta Cytol. 2011;55(1): 48-53.

16. Levi AW, Harigopal M, Hui P, Schofield K, Chhieng DC. Use of high-risk human papillomavirus testing in patients with low-grade squamous intraepithelial lesions. Cancer Cytopathol, 2011. 119(4):228-34.

17. Castle, PE, Fetterman B, Cox JT et al. The age-specific relationships of abnormal cytology and human papillomavirus DNA results to the risk of cervical precancer and cancer. Obstet Gynecol, 2010. 116(1):76-84.

18. Barron S, Austin RM, Li Z, Zhao C. Follow-up outcomes in a large cohort of patients with HPV-negative LSIL cervical screening test results. Am J Clin Pathol. 2015;143(4): 485-91.

19. Thrall MJ, Smith DA, Mody DR. Women $>$ or $=30$ years of age with low grade squamous intraepithelial lesion (LSIL) have low positivity rates when cotested for high-risk human papillomavirus: should we reconsider HPV triage for LSIL in older women? Diagn Cytopathol. 2010;38(6): 407-12. 